\title{
ARTICLE
}

\section{Experiment and analysis of neutron streaming in iron-polyethylene multi-layer shielding assembly}

\author{
Seiki Ohnishi ${ }^{a^{*}}$, Koichi Okuno ${ }^{\mathrm{b}}$, Akiko Konnai ${ }^{\mathrm{a}}$ and Kenichi Sawada ${ }^{\mathrm{a}}$ \\ ${ }^{a}$ National Maritime Research Institute, 6-38-1, Shinkawa, Mitaka-si, Tokyo, 181-0004, Japan; ${ }^{b}$ HAZAMA ANDO CORPORATION, \\ 515-1, Karima, Tsukuba-si, Ibaraki-ken, 305-0822, Japan
}

\begin{abstract}
In order to confirm accuracy of radiation transport codes, a neutron streaming experiment was conducted. An iron-polyethylene multi-layer shielding assembly was fabricated, and neutron dose distribution was measured. The shielding assembly has a three-legged rectangular duct of which the total length is $750 \mathrm{~mm}$. An encapsulated Cf-252 source was set in front of the entrance of the duct to irradiate the neutron detector, and the dose along the duct was measured by the bubble detectors placed inside. The neutron dose was also calculated by the Monte Carlo codes, PHITS and MCNP5, using various nuclear data libraries, JENDL-4.0, ENDF/B-VII.1 and JEFF-3.1. The difference depending on nuclear libraries is not significant. The calculations agree well with the experiments in both cases where PHITS and MCNP5 are used.
\end{abstract}

Keywords: streaming; Monte Carlo; MCNP; PHITS; bubble detector; neutron; JENDL-4.0; ENDF/B-VII.1; JEFF-3.1

\section{Introduction}

Multi-layer shielding which consists of hydrogen and medium-heavy nuclides is quite effective in a mixed neutron gamma radiation field, for example, transport casks [1] and interim storage facilities [2]. However, in the cases where cooling ducts or slits penetrate the shielding, neutron streaming occasionally becomes a problem [2]. Therefore, for the purpose of confirming calculation accuracy, comparison of experiments with calculations was designed.

PHITS [3], which can simulate particles and heavy ions transportation in three dimensions, was adopted to calculate neutron dose. There are many benchmark data in high energy region [4] because PHITS was originally developed for high energy particle transportation [5]. However, there are few benchmark studies in low energy region using nuclear data libraries. Therefore, validation experiment using fission neutrons is important. Furthermore, there is a work [6] which shows calculation results of PHITS differ from that of MCNP5 [7]. Therefore, MCNP5, which is widely used to calculate particle transportation, was also chosen for the sake of comparison. Three modern nuclear data libraries, JENDL-4.0 [8], ENDF/B-VII.1 [9], and JEFF-3.1 [10] were used in each calculation.

The aim of this study is validation of PHITS code and intercomparison of Monte Carlo codes for multi-layer shielding assembly having streaming structure like

*Corresponding author. Email: ohnishi@nmri.go.jp modular type interim storage facilities [2].

\section{Experiment}

\subsection{Geometry}

An iron-polyethylene multi layer shielding assembly with a three-legged rectangular duct was fabricated. Figure 1 shows the plan and side view of the assembly.

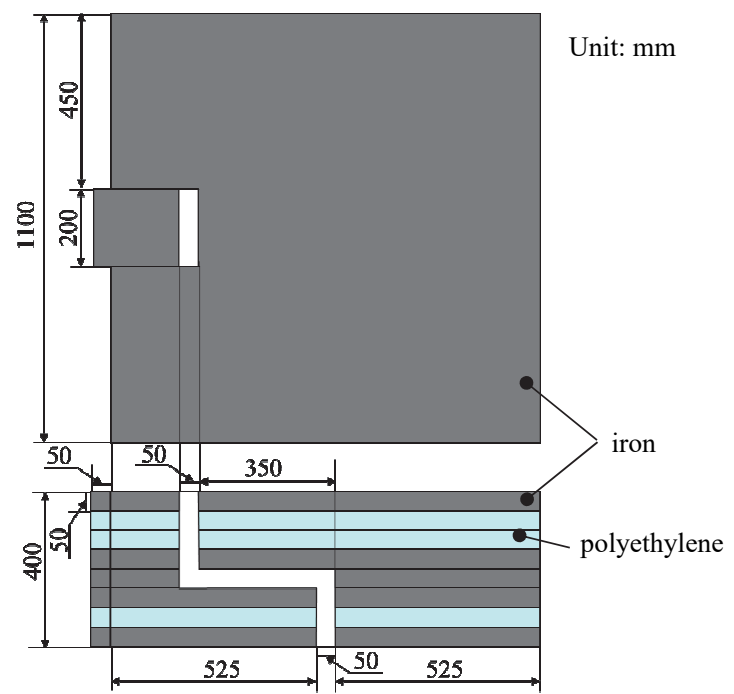

Figure 1. Plan view and side view of the shielding assembly.

This assembly is constructed with iron plates and 
polyethylene plates of which the length, width and thickness are $1100 \mathrm{~mm}, 1100 \mathrm{~mm}$, and $50 \mathrm{~mm}$ respectively. These plates have a rectangle notch, and the streaming duct is made by these notches and small plates. The cross section of the duct is $10,000 \mathrm{~mm}^{2}(50$ $\mathrm{mm} \times 200 \mathrm{~mm}$ ) and its whole length is $750 \mathrm{~mm}$.

\subsection{Neutron source}

An encapsulated Cf-252 spontaneous neutron source was used. A Cf oxide pellet is enclosed in an argon welding stainless capsule and an aluminum cylinder case. The source is stored in the bottom of a pit made with iron and polyethylene, and is lifted up to floor surface during irradiations. The encapsulation of the source is shown in Figure 2, and its arrangement within the experimental geometry is shown in Figure 3. The source neutron intensity was $2.37 \times 10^{7} \mathrm{~s}^{-1}$ at the time of the experiment.

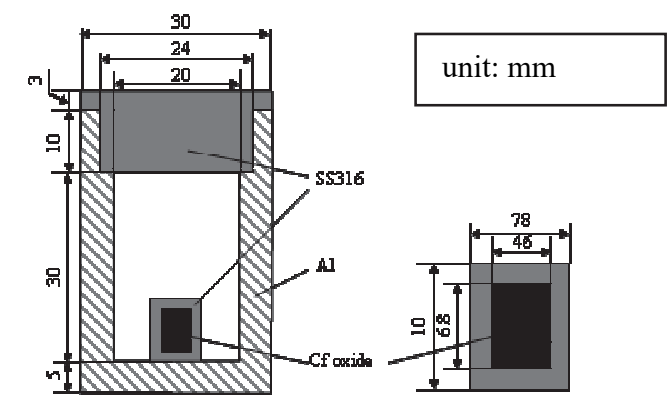

Figure 2. Source casing and capsule.

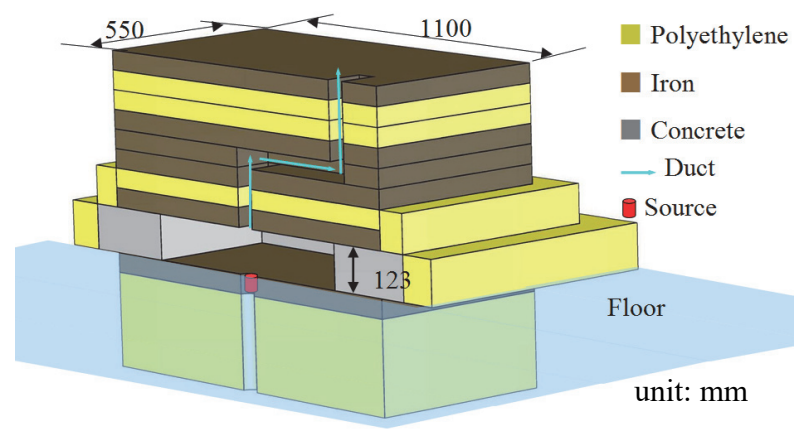

Figure 3. Sectional view of the assembly and neutron source.

\subsection{Detectors}

The size of neutron detector should be small enough to fit in the streaming duct. Therefore, bubble detectors (Bubble Technology Industries, BD100R-PND) were used for neutron detection.

The shape of BD100R-PND is a cylinder $120 \mathrm{~mm}$ long and $15 \mathrm{~mm}$ in diameter, and its sensitive volume is $15 \phi \times 45 \mathrm{~mm}$. Energy-dose response of the bubble detector [11] is similar to that of the effective dose [12]. These response curves are shown in Figure 4. Although BD100R-PND is a temperature compensation type, its response changes slightly with temperature. Then the irradiation room temperature was kept at 24 degrees Celsius, and the response of BD100R-PND increased by $10 \%$ from the nominal value in this environmental

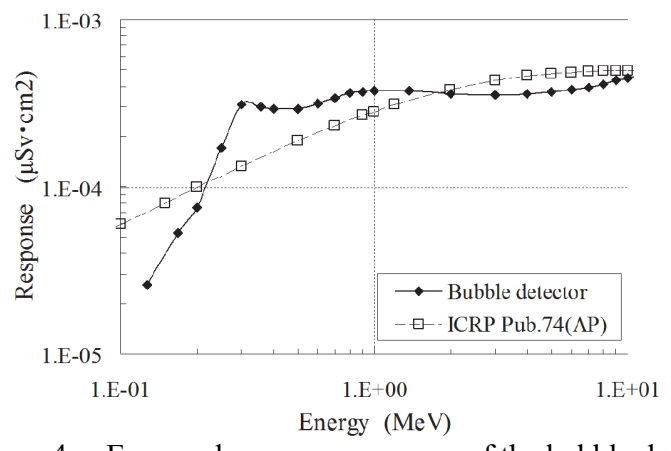

Figure 4. Energy-dose response curve of the bubble detector [11] and dose conversion coefficients [12].

condition [13].

Reading errors should be considered since the bubbles were counted by eye. To decrease such errors, average counts and standard deviations (reading errors) were obtained with the following procedures: we conducted neutron irradiation twice per each measuring point, and the number of bubbles were counted ten times for each detectors. In addition, because the process in which the bubble is generated is stochastic, the statistical error is estimated by the square root of the number of bubbles.

\section{Calculation}

The experimental geometry in Figure 1 was modeled three dimensionally. The calculation geometry model consists of the irradiation room, shielding assembly, and bubble detector. Figure 5 shows the irradiating room model. The thickness of the room wall was $1000 \mathrm{~mm}$, and typical concrete composition [14] was used.
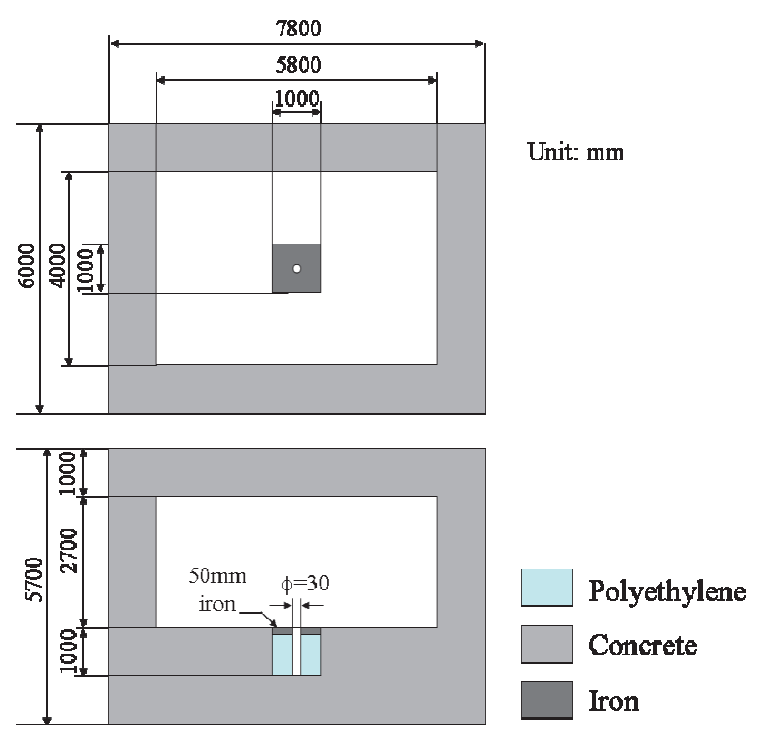

Figure 5. Irradiation room and source pit model.

The bubble detector was modeled as a $15 \phi \times 45 \mathrm{~mm}$ cylinder filled with polyacrylamide $\left(1.6 \mathrm{~g} / \mathrm{cm}^{3}\right)$. A track length estimator tally was set at the volume instead of pulse height tally because the leakage of secondary 
particles is negligible in bubble detectors. Not the effective dose conversion factor given in ICRP publication 74 but the response curve of the bubble detector shown in Figure 4 was applied in the Monte Carlo simulation for the purpose of comparison of the experiment with calculations.

The Maxwell fission energy spectrum was used as the energy distribution of neutron source for the comparison of MCNP and PHITS because PHITS does not support Watt fission spectrum. The source neutron spectrum $B$ is expressed as follows;

$$
B(E)=C E^{1 / 2} \mathrm{e}^{-E / T}
$$

where $E$ : energy, $C$ : normalization factor and $T$ : temperature $=1.42 \mathrm{MeV}$ for $\mathrm{Cf}-252$ spontaneous fission neutron source [15].

Dose distribution was calculated using PHITS and MCNP5. Three nuclear data libraries, JENDL-4.0, ENDF/B-VII.1, and JEFF-3.1 were tested for each code.

\section{Results and discussion}

The dose distribution was measured by the bubble detectors. To avoid mutual shielding of the detectors, only one detector was set in the duct at once on each irradiation duration. The results are listed in Table 1 and shown in Figure 6. Large numbers of bubbles lead to large reading errors. Therefore, irradiation duration was adjusted so that about 100 bubbles are generated whenever practicable. As a result, both the reading error and statistical error were around $10 \%$ in most measuring points.

Table 1. Measured dose and experimental error.

\begin{tabular}{ccccccc}
$\begin{array}{c}\text { Dist. } \\
(\mathrm{cm})\end{array}$ & $\begin{array}{c}\text { Dose } \\
(\mu \mathrm{Sv} / \mathrm{h})\end{array}$ & $\begin{array}{c}\text { Relative } \\
\text { error }\end{array}$ & & $\begin{array}{c}\text { Dist. } \\
(\mathrm{cm})\end{array}$ & $\begin{array}{c}\text { Dose } \\
(\mu \mathrm{Sv} / \mathrm{h})\end{array}$ & $\begin{array}{c}\text { Relative } \\
\text { error }\end{array}$ \\
\cline { 1 - 2 } 3.3 & $1.17 \mathrm{E}+4$ & 0.16 & & 48.3 & $4.27 \mathrm{E}+2$ & 0.09 \\
8.3 & $7.23 \mathrm{E}+3$ & 0.21 & & 53.3 & $2.86 \mathrm{E}+2$ & 0.10 \\
13.3 & $5.41 \mathrm{E}+3$ & 0.17 & & 58.3 & $1.59 \mathrm{E}+2$ & 0.08 \\
18.3 & $4.95 \mathrm{E}+3$ & 0.14 & & 63.3 & $8.00 \mathrm{E}+1$ & 0.10 \\
23.3 & $2.23 \mathrm{E}+3$ & 0.13 & & 68.3 & $3.41 \mathrm{E}+1$ & 0.13 \\
28.3 & $1.41 \mathrm{E}+3$ & 0.20 & & 73.3 & $2.45 \mathrm{E}+1$ & 0.17 \\
33.3 & $9.28 \mathrm{E}+2$ & 0.12 & & 78.3 & $1.44 \mathrm{E}+1$ & 0.20 \\
38.3 & $7.62 \mathrm{E}+2$ & 0.13 & & 83.3 & $8.23 \mathrm{E}+0$ & 0.25 \\
43.3 & $5.51 \mathrm{E}+2$ & 0.09 & & - & - & - \\
\hline
\end{tabular}

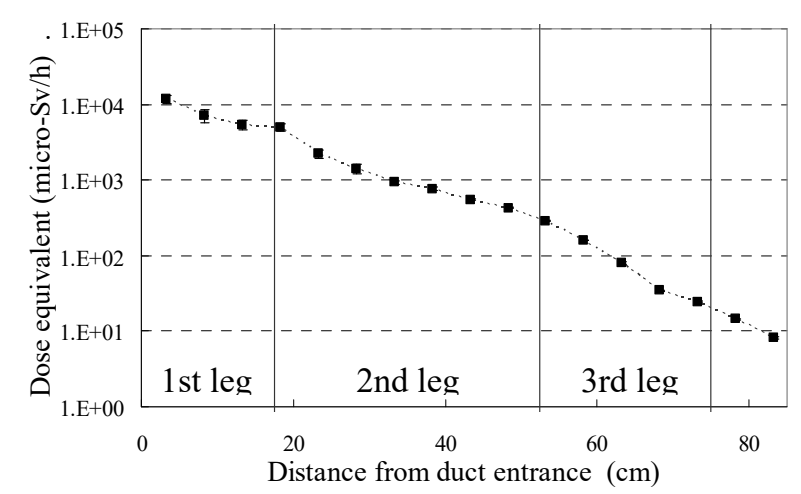

Figure 6. Neutron dose distribution along the duct.
The calculation results are compared with the measured doses in Figures 7 and 8 . The solid black lines in the figures represent range of $\mathrm{C} / \mathrm{E}$ within $1 \sigma$ experimental error described in Section 2.3. All the statistical errors of Monte Carlo calculation are less than $1 \%$, and omitted in the figures. It is remarked that the influence using different libraries is insignificant.

The Maxwellian energy spectrum was adopted in the calculations while the Watt spectrum was more adequate for Cf-252 spontaneous fission spectrum. However, it has been confirmed that the doses changed by only about $1 \%$ in the 1 st leg when the Watt fission spectrum source was used.

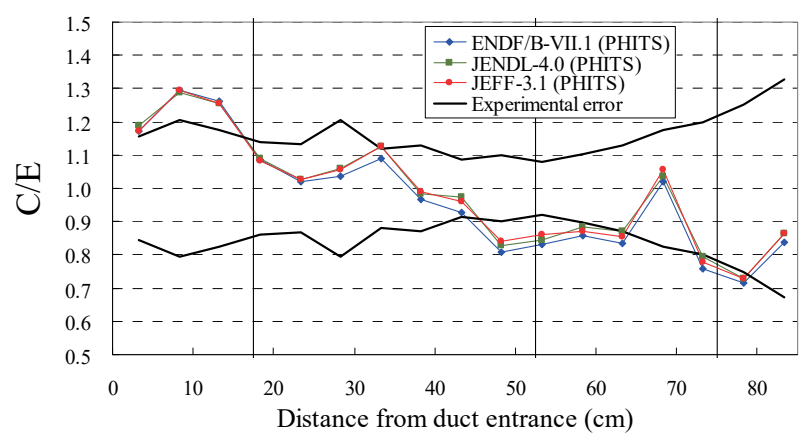

Figure 7. C/Es (Calculation/Experiment) by PHITS.

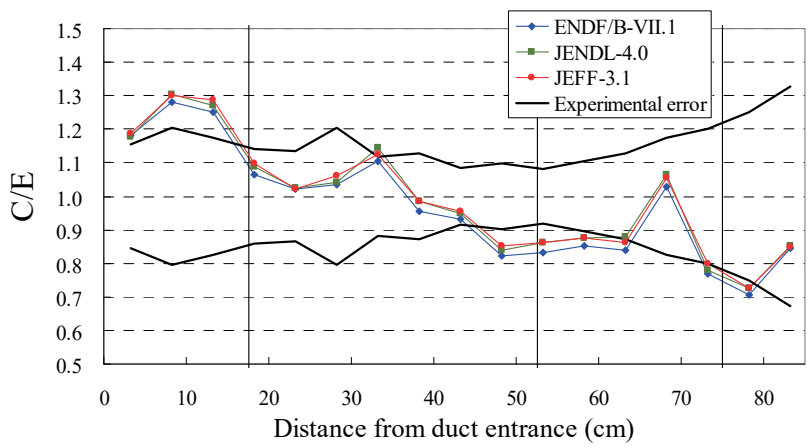

Figure 8. C/Es (Calculation/Experiment) by MCNP5.

The experimental errors become larger in the first-leg and third-leg, and $\mathrm{C} / \mathrm{Es}$ are apart from 1.0 there. The experimental errors increase in the point where the numbers of bubble are too many or too few. In the experiment, minimum irradiation duration is required because source capsule manipulation takes a certain time. Even though less sensitive detectors were set in the first leg, the number of bubbles and then its reading error became larger. Conversely, the number of bubbles became smaller around the exit of the duct, and thus the experimental statistical error was increased in the third-leg.

The discrepancy between calculation and experiment in the first-leg is also possibly caused by excessive amount of bubbles because the experimental doses are biased by counting losses of the bubbles. Moreover, the contribution of the neutrons scattered by the structures in the irradiation room are likely underestimated in the calculations because there exist other large equipments, for example racks and cranes, that are difficult to be 
modeled. This influence is significant in the third-leg because the doses there are relatively small. Therefore in the first-leg and 3rd-leg, the $\mathrm{C} /$ Es tend to deviate from 1.0. Both codes generally reproduce the experimental results within the range of 2-sigma, and $\mathrm{C} / \mathrm{E}$ is 0.7 even in the worst case.

Figure 9 shows the calculated dose ratios of PHITS to MCNP. In each calculation, the total number of histories was adjusted so that the fractional standard deviation (FSD) becomes 1\%. Therefore, the synthesized FSD of MCNP/PHITS ratio was $1.14 \%$ constantly. Figure 9 shows MCNP/PHITS ratios are oscillating within the range of synthesized FSD, that means, both results are statistically equivalent.

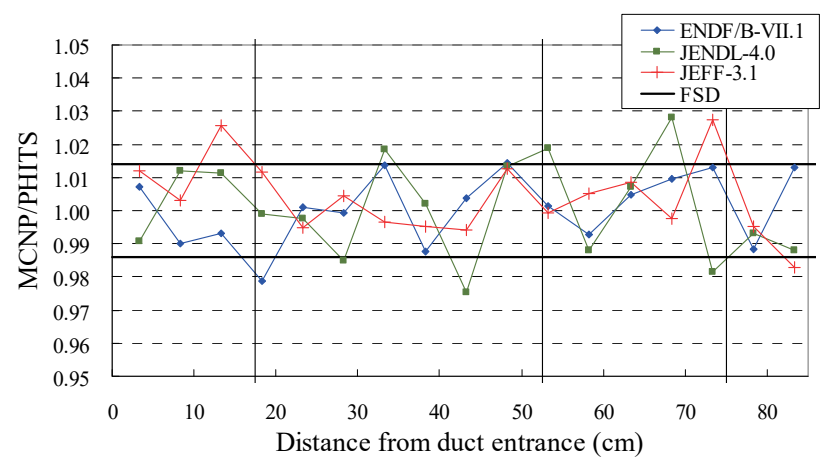

Figure 9. Calculated dose ratio, MCNP/PHITS.

\section{Conclusion}

Neutron dose distribution along the duct was measured in the multi-layer shielding assembly. The doses calculated by the Monte Carlo radiation transport codes and that measured by the bubble detectors agreed generally within the range of errors. Difference of nuclear data libraries had little influence on the results. PHITS code was also in agreement as well as the MCNP code.

\section{Acknowledgements}

The authors wish to thank Toshimasa Miura for the helpful advice on bubble detectors. Without the help of Naoteru Odano in planning and conducting the experiments, this work would not have been possible.

\section{References}

[1] The Federation of Electric Power Companies of Japan, FEPC - Return shipment of vitrified residues from France to Japan (5-2: Technical complements), http://www.fepc.or.jp/english/library/ about nuclear/france/05-2.html.

[2] M. Asami, S. Ohnishi, K. Kawakami, M. Matsumoto and N. Odano, Rational shielding ability evaluation for a modular type interim storage facility, Nihon-Genshiryoku-Gakkai Shi (J. At. Energy Soc. Jpn.) 6 (4) (2007), pp.460-475. [in Japanese]
[3] T. Sato, K. Niita, N. Matsuda, S. Hashimoto, Y. Iwamoto, S. Noda, T. Ogawa, H. Iwase, H. Nakashima, T. Fukahori, K. Okumura, T. Kai, S. Chiba, T. Furuta and L. Sihver, Particle and heavy ion transport code system PHITS, version 2.52, $J$. Nucl. Sci. Technol. 50:9 (2013), pp.913-923.

[4] Y. Iwamoto, T. Sato, S. Hashimoto, T. Ogawa, T. Furuta, S. Abe, T. Kai, N. Matsuda, R. Hosoyamada and K. Niita, Benchmark study of the recent version of the PHITS code, J. Nucl. Sci. Technol. 54 (2017), pp.617-635.

[5] H. Iwase, K. Niita and T. Nakamura, Development of general-purpose particle and heavy ion transport Monte Carlo code, J. Nucl. Sci. Technol. 39 (2002), pp.1142-1151.

[6] J.W. Shin, S. Bak, D. Kim, C.Y. Kim and S. Hong, GEANT4 and PHITS simulations of the shielding of neutrons from ${ }^{252} \mathrm{Cf}$ source, arXiv: $1402.1847 \mathrm{v} 2$ [physics.ins-det] (2014).

[7] X-5 Monte Carlo Team, MCNP - A General $N$-Particle Transport Code, Version 5 Volume I: Overview and Theory, LA-UR-03-1987 (2003, updated 2005).

[8] K. Shibata, O. Iwamoto, T. Nakagawa, N. Iwamoto, A. Ichihara, S. Kunieda, S. Chiba, K. Furutaka, N. Otuka, T. Ohsawa, T. Murata, H. Matsunobu, A. Zukeran, S. Kamada and J. Katakura, JENDL-4.0: A new library for nuclear science and engineering, J. Nucl. Sci. Technol. 48(1) (2011), pp.1-30.

[9] M.B. Chadwick, M. Herman, P. Obložinský, M.E. Dunn, Y. Danon, A.C. Kahler, D.L. Smith, B. Pritychenko, G. Arbanas, R. Arcilla, R. Brewer, D.A. Brown, R. Capote, A.D. Carlson, Y.S. Cho, H. Derrien, K. Guber, G.M. Hale, S. Hoblit, S. Holloway, T.D. Johnson, T. Kawano, B.C. Kiedrowski, H. Kim, S. Kunieda, N.M. Larson, L. Leal, J.P. Lestone, R.C. Little, E.A. McCutchan, R.E. MacFarlane, M. MacInnes, C.M. Mattoon, R.D. McKnight, S.F. Mughabghab, G.P.A. Nobre, G. Palmiotti, A. Palumbo, M.T. Pigni, V.G. Pronyaev, R.O. Sayer, A.A. Sonzogni, N.C. Summers, P. Talou, I. J. Thompson, A. Trkov, R.L. Vogt, S.C. van der Marck, A. Wallner, M.C. White, D. Wiarda and P.G. Young, ENDF/B-VII.1 Nuclear data for science and technology: cross sections, covariances, fission product yields and decay data, Nuclear Data Sheets 112 (2011), pp. 2887-2996.

[10]A. Koning, R. Forrest, M. Kellett, R. Mills, H. Henriksson and Y. Rugama, The JEFF-3.1 Nuclear Data Library, JEFF Report 21, OECD/NEA, Paris, (2006).

[11]Bubble Technology Industries, Data Sheet of Bubble Detectors, Revision 1 (2002).

[12]ICRP, Conversion coefficients for use in radiological protection against external radiation, ICRP Publication 74. Ann. ICRP 26 (1996), pp.3-4.

[13]H. Ing, Neutron measurements using bubble 
detectors -- terrestrial and space, Radiat. Meas. 33 (2001), pp. 275-286.

[14]Nuclear Safety Technology Center, Houshasenshisetu-No-Syaheikeisan-Jitsumumanyuaru (Practical Manual for Shielding Calculation in
Radiation Facility), (2007). [in Japanese]

[15]International Standard Organization, Reference neutron radiations Part 1: Characteristics and methods of production, ISO 8529-1:2001 (2001). 